\title{
Energy Balanced Routing Method for In-Network Data Aggregation in Wireless Sensor Networks
}

\author{
Juby K Baby P K Poonguzhali \\ Department of Electronics and Communication Engineering Hindusthan College of Engineering and \\ Technology, Coimbatore, Affiliated to Anna University \\ Department of Electronics and Communication EngineeringHindusthan college of engg \&technology, \\ Coimbatore Anna University Chennai
}

\begin{abstract}
A wireless sensor network (WSN) consists of a large number of wireless sensor nodes. Since wireless sensor nodes are battery powered devices, they have limited processing and transmission power. In order to transmit sensing data to receiver effectively, it is important to design a routing protocol for WSNs. Since energy conservation is a key issue in WSNs, data aggregation should be exploited in order to save energy. In this case, redundant data can be aggregated at intermediate nodes reducing the size and number of exchanged messages and, thus, decreasing communication costs and energy consumption. This paper propose an Enhanced Forward Aware Factor-Energy Balanced Routing Method (EFAF-EBRM) based on Data aggregation technique that has some key aspects such as a reduced number of messages for setting up a routing tree, maximized number of overlapping routes, high aggregation rate, and reliable data aggregation and transmission.. In the experiments, proposed method is compared with FAF-EBRM and LEACH. Experimental results show that proposed method outperforms FAF-EBRM and LEACH, which balances the energy consumption, prolongs the network function lifetime and provides the best aggregation quality .

Keywords: forward aware factor(FAF), Energy balance, Routing, Wireless sensor networks(WSNs).
\end{abstract}

\section{INTRODUCTION}

A Wireless Sensor Networks (WSNs) is a self-organization wireless network system constituted by numbers of micro sensors with limited energy. They are deployed to monitor the sensing field and collect information from physical or environmental condition and to co-operatively pass the collected data through the network to a main location. Due to the limited energy and communication ability of sensor nodes, it is important to design a network topology, routing algorithm and protocol for large-scale WSN communication system. Energy consumption is an important factor in system designs of WSNs. Traditionally, there are two approaches to accomplish the data collection task: Direct communication, and Multi-hop forwarding. In one hop wireless communication, the sensor nodes upload data directly to the sink, which may result in long communication distances and degrade the energy efficiency of sensor nodes. On the other hand, in multi-hop forwarding, data are transferred from the nodes to the sink through multiple relays, and thus communication distance is reduced[7]. However, since nodes closer to the sink have a much heavier forwarding load, their energy may be depleted rapidly, which degrades the network performance.

Clustering is an effective technique to reduce energy consumption in WSNs[13]. In clustering algorithm, a number of nodes in a network will be chosen as the cluster heads (CHs) and the remaining nodes will be regarded as the cluster members(CMs). CMs will form connections with the $\mathrm{CHs}$. A $\mathrm{CH}$ will collect data from its CMs. In WSN clustered hierarchical routing protocols, sometimes CMs are closer to the sink than $\mathrm{CH}$, but it should transmit data to $\mathrm{CH}$ first. This backward transmission result in waste of energy.

The aim of this project is to propose a new energy-balance routing protocol that uses forward transmission area(FTA) based on position of sink and final data flow direction. In other words, FTA define forward energy density which constitutes forward-aware factor with link weight, and propose a new communication protocol based on forward-aware factor,thus balancing the energy consumption and prolonging the network function lifetime[14],[16]

\section{Related Work}

\subsection{Low Energy Adaptive Clustering Hierarchy(LEACH) Protocol}

LEACH protocol is one of the most famous hierarchical routingprotocol for wireless sensor networks to increase the life time of network. In LEACH, all the nodes in a network organize themselves into local clusters. The protocol is divided into a setup phase when the clusters are organized and a steady state phase when $\mathrm{CH}$ receive data from all the CMs, perform data aggregationand transmit data to the remote base station. The operation of LEACH is illustrated in Fig1. In clustering algorithm, $\mathrm{CH}$ node is much more energy-intensive than a $\mathrm{CM}$. Thus, when a $\mathrm{CH}$ node in a cluster dies, all the $\mathrm{CMs}$ inside that cluster lose communication ability. 
LEACH allows randomized rotation of the high-energy cluster-head position such that it rotates among the sensors. In this way, the energy load associated with being a $\mathrm{CH}$ is evenly distributed among the sensing nodes. The $\mathrm{CH}$ node will know all the CMs in the cluster. Therefore it can create a TDMA schedule that informs each node exactly when to transmit its collected data

In the setup phase, each sensor node chooses a random number, $r$, between 0 and 1 . If this random number is less than a certain threshold value, $\mathrm{T}(\mathrm{n})$, the node becomes a $\mathrm{CH}$ for the current round.

Threshold value $T(n)$ is calculated by the equation,

$$
\mathrm{T}(\mathrm{n})=\underset{0}{\mathrm{p} /(1-\mathrm{p} \times \mathrm{r} \bmod (1 / \mathrm{p})) \quad, \quad \mathrm{n} \in \mathrm{G}}
$$

This equation incorporates the desired percentage to become a $\mathrm{CH}$, the current round, and the set of nodes that have not been selected as a $\mathrm{CH}$ in the last $(1 / \mathrm{P})$ rounds where $\mathrm{p}$ is cluster head probability. If random number is less than $\mathrm{T}(\mathrm{n})$, the node will broadcasts an advertisement message (ADV) as the $\mathrm{CH}$. This message contains the node's ID and a header that distinguishes this message as an announcement message. Each noncluster-head selects the $\mathrm{CH}$ that requires the minimum communication energy, based on the received signal strength of the advertisement from each $\mathrm{CH}$. After the selection of $\mathrm{CH}$, each node must inform thecluster-head node that it will be a member of the cluster. Each node sends a join-request message (Join-REQ) back to the selected $\mathrm{CH}[2],[6]$.

In the steady-state phase, operation is broken into frames where nodes transmit their data to the $\mathrm{CH}$ at most once per frame during their allocated transmission slot. To reduce energy dissipation, each CM sets the amount of transmission power by using power control. It is based on the received strength of the cluster-head advertisement. The $\mathrm{CH}$ receives all the data from the nodes in the cluster by keeping its receiver on and then the resultant data are sent from the $\mathrm{CH}$ to the base station.

\subsection{Forward Aware Factor-Energy Balanced Routing Method(FAF-EBRM)}

In WSN clustered hierarchical routing protocol, sometimes cluster members in a cluster are nearer to the sink than the $\mathrm{CH}$, but it should transmit data to $\mathrm{CH}$ first. It results backward transmission of data and thus leads to waste of energy.

In this method, an energy-balanced routing protocol is designed that uses forward transmission area(FTA) based on position of sink and final data flow direction. In other words, FTA define forward energy density which constitutes forward-aware factor with link weight, and propose a new communication protocol based on forward-aware factor, thus balancing the energy consumption and prolonging the network function lifetime[3].

\section{Enhanced Forward Aware Factor-Energy Balanced Routing Method}

This paper propose an Enhanced Forward Aware Factor-Energy Balanced Routing Method (EFAF-EBRM) based on Data aggregation technique that has some key aspects such as a reduced number of messages for setting up a routing tree, maximized number of overlapping routes, high aggregation rate, and reliable data aggregation and transmission. According to data transmission mechanism of WSN, we quantify the forward transmission area ,define forward energy density which constitutes forward-aware factor with link weight. For energy efficient transmission in event-driven WSN, Data should be reduced. It requires proper routing method for reliable transmission of aggregated data to sink from the source nodes. This paper propose a new communication protocol based on forward-aware factor in order to determine next-hop node and Data Routing for In-Network aggregation(DRINA) protocol to reduce the number of transmissions and thus balancing the energy consumption, prolonging the network function lifetime and to improve QoS of WSN.

\subsection{Description of Network model}

Consider a rectangular sensing field $\mathrm{W} \times \mathrm{H}$ in which sensor nodes are randomly distributed as shown in Fig 1. Regional central node( cluster head) receives data from all the CMs and then transfers tothe sink node(sink). 


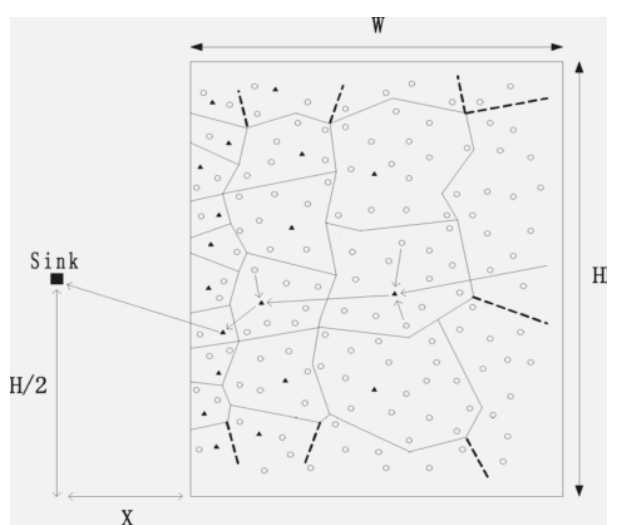

Fig 1. Map of sink and sensor node distribution

The descriptions of network model are given below

1) All sensor nodes are isomorphic and they have limited energy and communication ability. Here, identifier for a node is represented as ' $\mathrm{i}$ '.

2) $\mathrm{CH}$ is more energy intensive than a $\mathrm{CM}$. Thus, when a $\mathrm{CH}$ in a cluster dies, all the $\mathrm{CMs}$ inside that cluster lose communication ability. However, addition of energy of sink node is possible. But, it is not possible to change the location of nodes and sink after being fixed.

3) According to distance to receiver, nodes can vary its transmission power. The sink node can broadcast advertisement message to all nodes in the sensing field. The distance between source and receiver canbe determined by received signal strength. When the data transmission distance is more than certain threshold $\mathrm{d} 0$, the energy consumption would rise sharply. The threshold value is given by,

$$
d_{0}=\sqrt{\varepsilon_{f s}^{2} / \varepsilon_{m p}}
$$

where, $\varepsilon_{f s}$ and $\varepsilon_{m p}$ are the energy coefficients.

\subsection{Establishment of the Network Model}

Let FTA(i) be the forward transmission area of node(i), N(i) be the set of nodes that have communication link with node (i), N'(i) be the set of nodes of N(i) that have edge with node $\mathrm{i}, d_{i j}$ be the distance between node $\mathrm{i}$ and node j. Consider a circle $\odot_{1}$ with sink as the centre and another circle $\odot_{2}$ with node, $\mathrm{i}$ as the centre and $d_{i p}$ as the radius as shown in Fig 2.

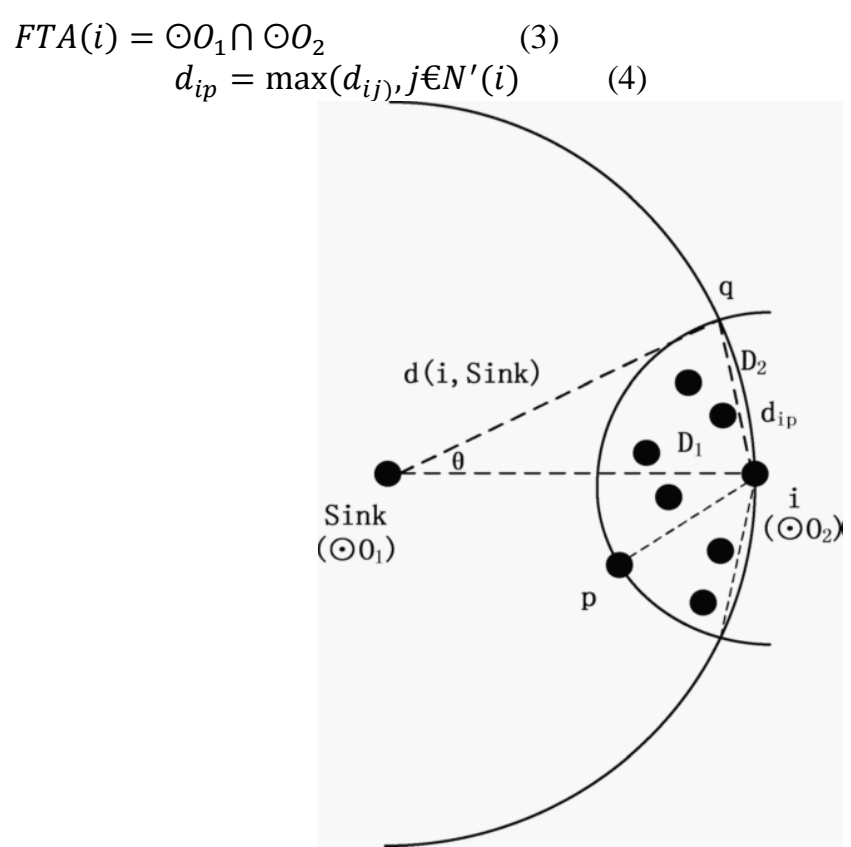

Fig 2. Forward Transmission Area of node i 
This paper proposes an energy balanced routing protocol that uses forward transmission area (FTA) based on position of sink and final data flow direction. In fig 2 , the arc of circle $\odot_{1}$ shows the possibility of backward transmission of node i. Circle $\odot_{2}$ contains all nodes that directly connected with node $\mathrm{i}$. These 2 circles contains all possiblenext nodes under topology and routing algorithm.FTA(i) is the overlap section of these 2 circles.

By using (4), the area of FTA(i) is $S_{F T A}(i)$ and can be written as

$$
\left(2 / 3 \prod-\frac{\sqrt{3}}{2}\right)\left[\operatorname{maxd}_{i j}\right]^{2} \leq S_{F T A}(i)<\frac{1}{2} \Pi\left[\max \left(d_{i j}\right)\right]^{2}
$$

Let $\mathrm{d}(\mathrm{i}$, sink $)=d_{1}, d_{i p}=\max \left(d_{i j}\right)=d_{2}$. According to cosine theorem, angle $\mathrm{\theta}$ can be written as

$$
\Theta=\operatorname{arcos}\left[1-\frac{1}{2}\left(\frac{d_{2}}{d_{1}}\right)^{2}\right]
$$

Consider a case in which sink is a neighbour of node $\mathrm{i}$ as shown in Fig 3 . In this case, $d_{1}$ become equal to $d_{2}$. If $d_{1}=d_{2}$, then minimum area of FTA(i) becomes $\left(\frac{2 \prod}{3}-\frac{\sqrt{3}}{2}\right) d_{2}^{2}$

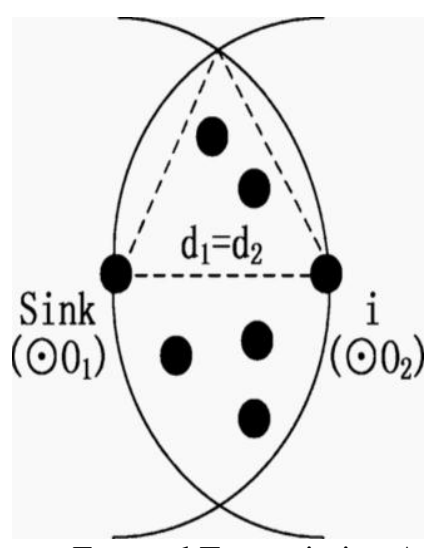

Fig 3.Minimum Forward Transmission Area of node i

Consider an another case in which $d_{1}$ tends to infinity as shown in Fig 4. In this case, the maximum area of FTA(i) is $\prod d_{2}^{2} / 2$. When the radius of $\odot_{1}$ become infinity, its arc tends to be a straightline. This arc passes the centre of $\odot_{2}$ and approximately dividing $\odot_{2}$ equally. The area of FTA(i) satisfies the condition of inequality

$$
0<S_{F T A}(\mathrm{i})<\frac{1}{2} \Pi d_{0}^{2}
$$

Where $d_{0}$ is the communication radius

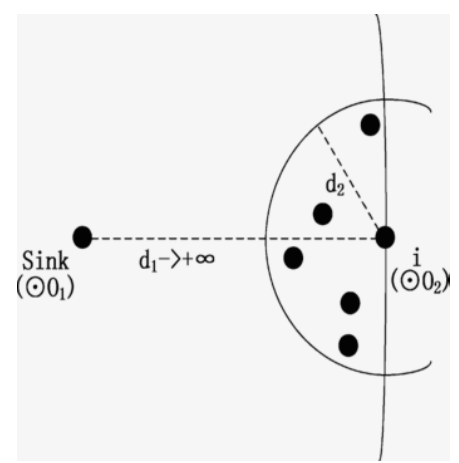

Fig 4.Maximum Forward Transmission Area of node i

The node's forward energy density $\mathrm{FED}(\mathrm{i}, \mathrm{t})$ satisfies thecondition of equality.

$$
F E D(i, t)=\frac{\sum_{j € F T A(i)} E_{j}(t)}{S_{F T A}(i)}
$$


Where $E_{j}(t)$ is the energy of node jat time $\mathrm{t}$ and the numerator in (8) represents energy of all the neighbour combined in function FTA(i). The forward aware factor (FAF) of the transmission link between node $i$ and node $\mathrm{j}$ can be written as

$$
F A F(i j)=\propto \frac{F E D(j)}{\sum_{j € F T A(i)} F E D(j)}+\beta \frac{w_{i j}}{\sum_{j € F T A(i)} w_{i j}}
$$

In the above equation, the first term represents the FED of all possible next hop nodes that means the ability to transmit data. The second term represents the weight of transmit link which is used to select next hop node directly. In (9), the denominator of the first term represents FAF of all the neighbours combined in FTA(i) and that of second term represents all of the link weights combined that $i$ has in FTA. $\propto$ and $\beta$ are positive harmonic coefficients and they are related by

$$
\propto+\beta=1
$$

\subsection{Design of the Enhanced Forward Aware Factor-Energy Balanced Routing Method (FAF-EBRM)}

Large scale WSN uses FAF-EBRM for static data collection and event detection[1]. The routing algorithm of this method is given below.

1) Determine FTA(i) and all of the possible next-hop nodes of node i . First, take as the communication radius, determine the set of all of the nodes that have edges with i, N'(i).Select the nodes that closer to Sink than i does, which constitute the set of all of the possible next-hop nodes and the furthest node determine FTA(i).

2) Determine FTA(j)and $S_{F T A}(j)$ of each possible next-hopnode. Determine FTA(j) as we determined FTA(i) .

3) Calculate FED(j) of each possible next-hop node according to (8).

4) Calculate the weight of edges between $i$ and each nodes.

5) Calculate FAF of each possible transmit link by using (9). Choose the next-hop node using the following equation

$$
j=\max _{j}[F A F(i j)]
$$

6) If there is no node closer to Sink than i in N'(i), directly compare FAF of all of the nodes in N'(i), and choose the next-hop node according to (10). If there is no node in N'(i), i will increase the transmit power to get a longer radius than $d_{0}$ until connected with another node, or i will abandon the packet.

7) If Sink is among the forward transmit nodes, $i$ will transmit

data directly to Sink and accomplish the procedure[11].

For energy efficient transmission in event-driven WSN, Data should be reduced. It requires proper routing method for reliable transmission of aggregated data to sink from the source nodes. This paper propose a new communication protocol based on forward-aware factor in order to determine next-hop node and Data Routing for In-Network aggregation(DRINA) protocol to reduce the number of transmissions and thus balancing the energy consumption, prolonging the network function lifetime and to improve QoS of WSN.

\subsubsection{Data Routing for In-Network Aggregation(DRINA)}

The main goal of the DRINA algorithm is to build a routing tree with the shortest paths that connect all source nodes to the sink while maximizing data aggregation. This algorithm considers the following roles in the routing infrastructure creation:

1. Collaborator: A node that detects an event and reports the gathered data to a coordinator node.

2. Coordinator: A node that also detects an event and is responsible for gathering all the gathered data sent by collaborator nodes, aggregating them and sending the result toward the sink node.

3. Sink: A node interested in receiving data from a set of coordinator and collaborator nodes.

4. Relay: A node that forwards data toward the sink.

The DRINA algorithm can be divided into three phases. In Phase 1, the hop tree from the sensor nodes to the sink node is built. In this phase, the sink node starts building the hop tree that will be used by Coordinators for data forwarding purposes. Phase 2 consists of cluster formation and cluster-head election among the nodes that detected the occurrence of a new event in the network. Finally, Phase 3 is responsible for both setting up a new route for the reliable delivering of packets and updating the hop tree. 


\subsubsection{Phase 1: Building the Hop Tree}

In this phase, the distance from the sink to each node is computed in hops. This phase is started by the sink node sending, by means of a flooding, the Hop Configuration Message (HCM) to all network nodes. The HCM message contains two fields: ID and HopToTree, where ID is node identifier that started or retransmitted the HCM message and HopToTree is the distance, in hops, by which an HCM message has passed. The HopToTree value is started with value 1 at the sink, which forwards it to its neighbors (at the beginning, all nodes set the HopToTree as infinity). Each node, upon receiving the message HCM, verifies if the value of HopToTree in the HCM message is less than the value of HopToTree that it has stored and if the value of First Sending is true. If that condition is true then the node updates the value of the NextHop variable with the value of the field ID of message HCM, as well as the value of the HopToTree variable, and the values in the fields ID and HopToTree of the HCM message. The node also relays the HCM message.Otherwise, if that condition is false, which means that the node already received the HCM by a shorted distance, then the node discards the received HCM message .The steps described above occur repeatedly until the whole network is configured. Before the first event takes place, there is no established route and the HopToTree variable stores the smallest distance to the sink. On the first event occurrence, HopToTree will still be the smallest distance; however, a new route will be established. After the first event, the HopToTree stores the smaller of two values: the distance to the sink or the distance to the closest already established route.

\subsubsection{Cluster Formation}

When an event is detected by one or more nodes, the leader election algorithm starts and sensing nodes will be running for leadership (group coordinator).For this election, all sensing nodes are eligible. If this is the first event, the leader node will be the one that is closest to the sink node. Otherwise, the leader will be the node that is closest to an already established route. In the case of a tie, i.e., two or more concurrent nodes have the same distance in hops to the sink (or to an established route), the node with the smallest ID maintains eligibility. Another possibility is to use the energy level as a tiebreak criterion. At the end of the election algorithm only one node in the group will be declared as the leader (Coordinator). The remaining nodes that detected the same event will be the Collaborators. The Coordinator gathers the information collected by the Collaborators and sends them to the sink. A key advantage of this algorithm is that all of the information gathered by the nodes sensing the same event will be aggregated at a single node (the Coordinator), which is more efficient than other aggregation mechanisms (e.g., opportunistic aggregation).

\subsubsection{Routing Formation and Hop Tree Updates}

The elected group leader starts establishing the new route for the event dissemination. For that, the Coordinator sends a route establishment message to its Next Hop node. When the Next Hop node receives a route establishment message, it retransmits the message to its Next Hop and starts the hop tree updating process. These steps are repeated until either the sink is reached or a node that is part of an already established route is found. The routes are created by choosing the best neighbour at each hop. The choices for the best neighbour are twofold: 1) when the first event occurs, the node that leads to the shortest path to the sink is chosen (Fig. $5 \mathrm{a}$ ); and $5 \mathrm{~b}$ ) after the occurrence of subsequent events, the best neighbour is the one that leads to the closest node that is already part of an established route (Fig. 5c).

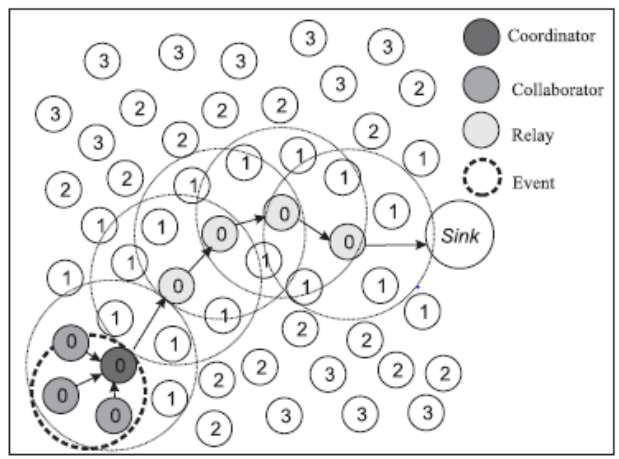

(a)Example of the routing tree to 1 event 


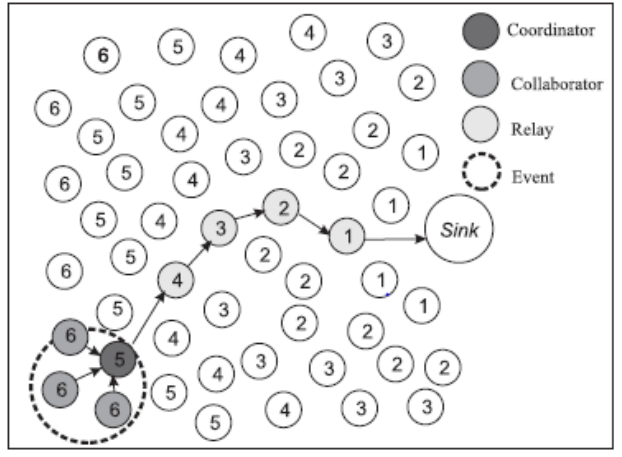

(b) Update of the hop tree

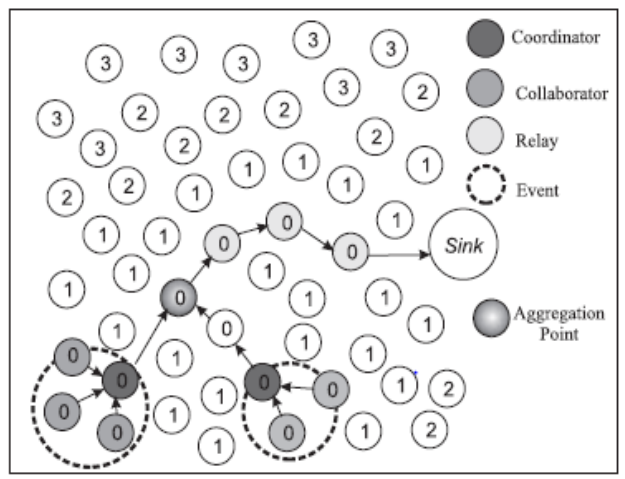

(c)Example of the routing tree to event

Fig.5. Example of establishing new routes and updating the hop tree.

\section{Performance Evaluation}

In this section, we evaluate the proposed EFAF-EBRM algorithm and compare its performance to two other known routing protocols: the FAF-EBRM and LEACH algorithms. These two algorithms were chosen for being well known in the literature and have the same goals that the proposed EFAF-EBRM algorithm. The default simulation parameters are presented in Table 1. For each simulation set, a parameter shown in Table 1 will be varied as described in the evaluated scenario.

TABLE 1

Simulation Parameters

\begin{tabular}{c|c}
\hline Parameter & Value \\
\hline \hline Sink node & 1 (top left) \\
Network size & 1024 \\
Communication radius (m) & 80 \\
\# of events & 3 \\
Event radius (m) & 50 \\
Event duration (hours) & 3 \\
Loss probability (\%) & 0 \\
Simulation duration (hours) & 4 \\
Notification interval $(\mathrm{sec})$ & 60 \\
Sensor field $\left(\mathrm{m}^{2}\right)$ & $700 \times 700$ \\
Node density (node $\left./ \mathrm{m}^{2}\right)$ & 21.7
\end{tabular}

We evaluate the EFAF-EBRM performance under the following metrics:

1. Data packet delivery rate: Number of packets that reach the sink node. This metric indicates the quality of the routing tree built by the algorithms - the lower the packet delivery rate, the greater the aggregation rate of the built tree

2. Control packet overhead: Number of control messages used to build the routing tree including the overhead to both create the clusters and set up all the routing parameters for each algorithm.

3. Efficiency (packets per processed data): It is the rate between the total packets transmitted (data and control packets) and the number of data received by the sink.

4. Routing tree cost: Total number of edges in the routing tree structure built by the algorithm.

5. Loss of raw data.

6. Loss of aggregated data:Number of aggregated data packets lost during the routing. In this metric, if a packet contains $\mathrm{X}$ aggregated packets and if this packet is lost, it is accounted the loss of $\mathrm{X}$ packets. 
7. Transmissions number.

8. Number of transmissions: Sum of control overhead and data transmissions, i.e., the total packets transmitted.

9. Number of Steiner nodes: Number of Steiner nodes in the routing structure, i.e., the number of relay nodes.

Table 2 shows the basic characteristics of LEACH, FAF-EBRM and EFAF-EBRM.

TABLE 2

Summary of the Basic Characteristics of Assessed Algorithms

\begin{tabular}{|l|l|l|l|l|l|l|l|}
\hline Scheme & EBF & NLN & PRR & PDR & Overhead & Throughput & Efficiency \\
\hline LEACH & Low & Low & Very low & Low & Very high & Low & Low \\
\hline FAF-EBRM & High & High & High & High & High & High & Medium \\
\hline EFAF-EBRM & High & High & High & Very high & Medium & Very high & high \\
\hline
\end{tabular}

\section{Simulation Results}

Here, we compare LEACH and EFAF-EBRM by three parameters: energy-balanced factor (EBF), number of last- surviving nodes (NLN) and function lifetime (FL), packets reception radio (PRR). To measure the balance of energy consumption of routing protocols, EBF is defined as the standard deviation of all the nodes' residual energy

$$
\left.E B F=\sqrt{\left(\frac{1}{N}\right.} \sum_{i=1}^{N}\left[E_{i}(t)-E_{a v g}(t)\right]^{2}\right)
$$

Where $\mathrm{N}$ is the number of the whole network nodes, $E_{i}(t)$ is the residual energy of node at time and $E_{\text {avg }}(t)$ is the average value of the residual energy of all of the nodes.

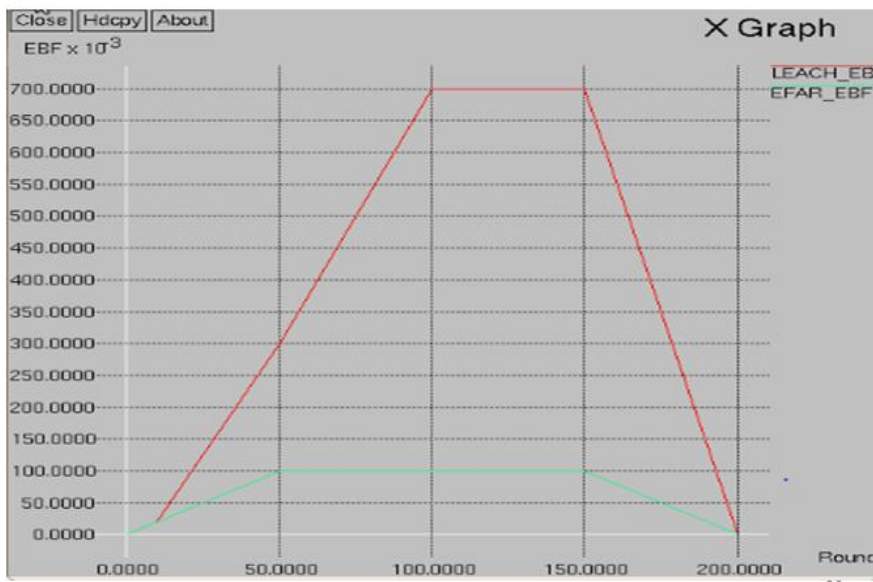

Figure 6: Simulation showing comparison of Energy Balanced Factor $(\mathrm{EBF})$

FL is directly related to NLN; the definition and requirement of FL is different under different conditions, as some require no death node, and some can still work when a certain percentage of nodes still work. In our experiment, we consider two conditions of FL, one is the time from the network begins to the first death of the nodes, another is the time from the network begin to half the nodes dead.

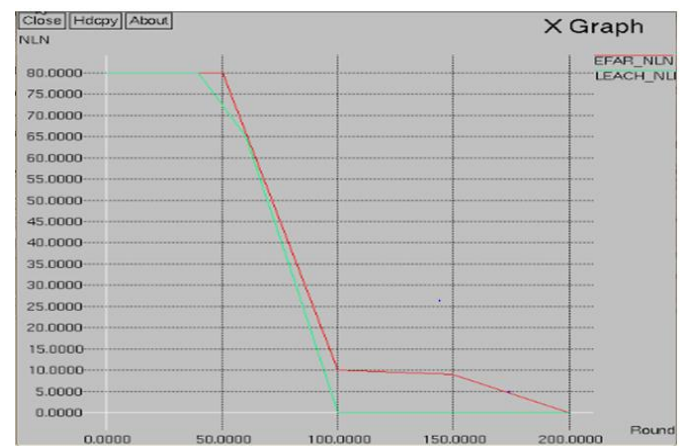

Figure 7: Simulation showing comparison of Number of Last-surviving Nodes (NLN) 
PRR means the ratio of the data that sink actually received to the data that sink is supposed to be received. PRR can measure WSN work situation intuitively.

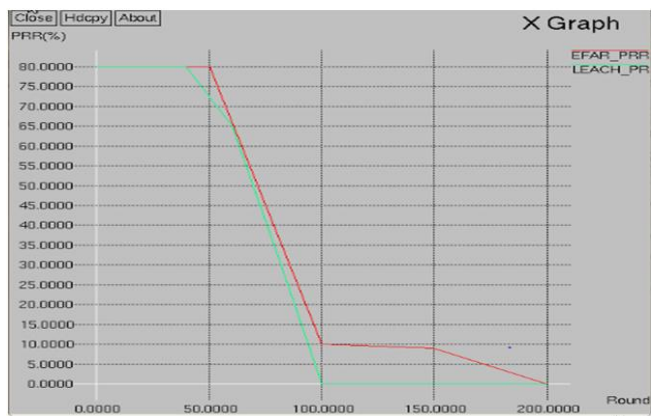

Figure 8: Simulation showing comparison of Packet Reception Radio(PRR in \%)

To compare two protocols conveniently and intuitively,

EFAF-EBRM also uses round as the time scale. Figs. 6-8 shows that the EBF, NLN, and PRR of three protocols in 200 rounds experiments. In Fig. 6, the EBF of FAF-EBRM increase slightly at first and keep a stable situation, then increase a little time, and return to 0 as the energy of the whole network is using up. In Fig. 7, the first death of FAF-EBRM node turns up until Round 200, and the procedure of the nodes' death is fast and late. In Fig. 8, the PRR of FAF-EBRM keeps $100 \%$ ratio for 200 rounds, and the decline stage accounting for a small proportion.

These three parameters of EFAF-EBRM is same as that of FAF-EBRM. But, in addition to these parameters, proposed method have some other advantages. The additional advantages of EFAF-EBRM over existing methods are given below

- Decreases communication overhead

- Increases throughput and Packet delivery ratio

Communication overhead means the ratio of routing packet to the received packet

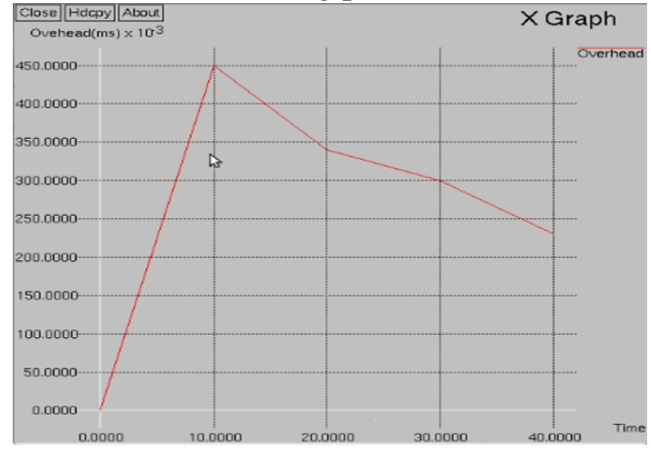

Figure 9: Simulation showing communication overhead versus time of EFAF-EBRM

Packet Delivery Ratio (PDR) means the ratio of the received packet to the Generated packet

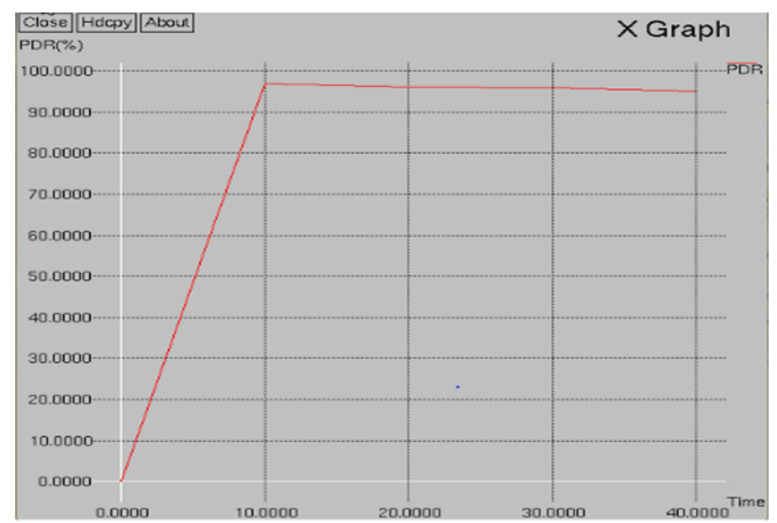

Figure 10: Simulation showing Packet Delivery Ratio versus Time of EFAF-EBRM 
From the above results, we can see that EFAF-EBRM has a higher performance than LEACH and FAFEBRM, which balances the energy consumption, prolongs the function lifetime and guarantees high QoS (such as Energy-Balanced, Long-Surviving, Packets Reception Radio) of WSNs.

\section{Conclusions And Future Enhancements}

Energy balanced routing algorithms play an important role in event-based WSNs. In this work, we presented the EFAF EBRM based on Forward Aware Factor and Data Agregation algorithm. Our proposed EFAF-EBRM algorithm was extensively compared to two other known routing algorithms, the FAF-EBRM and $\mathrm{LEACH}$, regarding scalability, communication costs, delivery efficiency, aggregation rate, and aggregated data delivery rate. By maximizing the aggregation points and offering a fault tolerant mechanism to improve delivery rate, the obtained results clearly show that EFAF-EBRM outperformed the FAF-EBRM and LEACH algorithms for all evaluated scenarios. Also, we show that our proposed algorithm has some key aspects required by WSNs aggregation aware routing algorithms such as a reduced number of messages for setting up a routing tree, maximized number of overlapping routes, high aggregation rate, and reliable data aggregation and transmission. As future work, spatial and temporal correlation of the aggregated data will also be taken into consideration as well as the construction of a routing tree that meets application needs. We also plan to modify the Proposed algorithm to stochastically select nodes that will be part of the communication structure. The goal is to find a balance between the overhead and the quality of the routing tree. In addition, new strategies will be devised to control the waiting time for aggregator nodes based on two criteria: average distance of the event coordinators, and spatial and semantics event correlation.

\section{Acknowledgment}

Prof. Dr V S Jayanthi, HOD of the Electronics and Communication Engineering department who followed the missions of ISRO for a long time, has contributed enormously to this paper and my research. His support during my work was incredibly invaluable. We would like to thank Assist prof. Ms.P K Poonguzhali of the Electronics and Communication Engineering department who provided a precious support to our work with her careful and knowledge able revision of the manuscript.

\section{References}

[1] A. L. Barabási, "Scale-free networks: A decade and beyond," Science, vol. 325, no. 5939, pp. 412-413, 2009

[2] D. G. Zhang and X. D. Zhang, "Design and implementation of embedded un-interruptible power supply system (EUPSS) for web-based mobile application," Enterprise Inf. Syst., vol. 6, no. 4, pp. 473-489, 2012.

[3] W. B. Heinzelman, A. P. Chandrakasan, and H. Balakrishnan, "An application- specific protocol architecture for wireless sensor networks," IEEE Trans. Wireless Commun., vol. 1, no. 4, pp. 660-670, Oct. 2002.

[4] I. K. Samaras and G. D. Hassapis, "A modified DPWS protocol stack for 6LoWPAN-based wireless sensor networks," IEEE Trans. Ind. Inf., vol. 9, no. 1, pp. 209-217, Feb. 2013.

[5] D.G. Zhang, "Anew approach and system for attentivemobile learning based on seamless migration," Appl. Intell., vol. 36, no. 1, pp. 75-89, 2012 .

[6] D. G. Zhang, "Web-based seamless migration for task-oriented nomadic service," Int. J. Distance E-Learning Technol., vol. 4, no. 3, pp. $108-115,2006$

[7] Q. J. Chen, S. S. Kanhere, andM. Hassan, "Analysis of per-node traffic load in multi-hop wireless sensor networks," IEEE Trans. Wireless Commun., vol. 8, no. 2, pp. 958-967, Apr. 2009.

[8] J. Jin et al., "Handling inelastic traffic in wireless sensor networks," IEEE Trans. Sel. Areas Commun., vol. 28, no. 7, pp. 1105-1115, Jul. 2010.

[9] D. G. Zhang and X. J. Kang, “A novel image de-noising method based on spherical coordinates system,” EURASIP J. Adv. Signal Process., vol. 1, pp. 110-117, 2012

[10] X. Li and G. R. Chen, “A local-world evolving network model,” Physica A, vol. 328, no. 1-2, pp. 274-286, 2003.

[11] A. S. Ruelaet al., "Evolutionary design of wireless sensor networks based on complex networks[A]," in Proc. 5th Int. Conf. Intell. Sensors, Sensor Netw., Melbourne, Victoria, Australia, 2009, pp. 237-242.

[12] J. Aweya, "Technique for differential timing transfer over packet networks," IEEE Trans. Ind. Inf., vol. 9, no. 1, pp. 325-336, Feb. 2013.

[13] C. T. Cheng, C. K. Tse, and F. C. M. Lau, "A clustering algorithm for wireless sensor networks based on social insect colonies," IEEE Sensors J., vol. 11, no. 3, pp. 711-721, Mar. 2011.

[14] H. B. Zhang and H. Shen, "Energy-efficient beaconless geographic routing in wireless sensor networks," IEEE Trans. Parallel Distrib. Syst., vol. 21, no. 6, pp. 881-896, Jun. 2010.

[15] S. Vural and E. Ekici, "On multihop distances in wireless sensor networks with random node locations," IEEE Trans. Mobile Computing, vol. 9 , no. 4, pp. 540-552, Apr. 2010

[16] F. V. C. Martins and E. G. Garrano, "A hybrid multiobjective evolutionary approach for improving the performance of wireless sensor networks," IEEE Sensors J., vol. 11, no. 3, pp. 545-554, Mar. 2011.

[17] P. Erdös and A. Rényi, "On the evolution of random graphs," Math. Inst. Hungarian Acad. Sci., vol. 5, pp. 60-67, 1960

[18] D. J. Watts and S. H. Strogatz, "Collective dynamics of small-world networks," Nature, vol. 393, no. 6684, pp. 440-442, 1998.

[19] G. Anastasi, M. Conti, M. Francesco, and A. Passarella, "Energy Conservation in Wireless Sensor Networks: A Survey," Ad Hoc Networks, vol. 7, no. 3, pp. 537-568, May 2009.

[20] H.S. AbdelSalam and S. Olariu, "A Lightweight Skeleton Construction Algorithm for Self-Organizing Sensor Networks,"pp. 1-5, 2009.

[21] L. Villas, A. Boukerche, R.B. de Araujo, and A.A.F. Loureiro,"Highly Dynamic Routing Protocol for Data Aggregation in Sensor Networks," pp. 496-502, 2010. 